\title{
GROUPS WITHOUT PROPER ISOMORPHIC QUOTIENT GROUPS
}

\author{
REINHOLD BAER
}

If $f$ is a homomorphism of the group $G$, and if $g$ is an isomorphism of the image group $G^{f}$, then $f g$ is a homomorphism of $G$ too; and this homomorphism is an isomorphism if, and only if, $f$ is an isomorphism. Consequently the following three properties of the group $G$ imply each other.

(1) Homomorphisms of $G$ upon isomorphic groups are isomorphisms.

(2) Homomorphisms of $G$ upon itself are isomorphisms.

(3) If $N$ is a normal subgroup of $G$ such that $G$ and $G / N$ are isomorphic, then $N=1$.

Groups meeting these requirements (1) to (3) shall be termed $Q$-groups. A direct product of an infinity of isomorphic groups different from 1 is certainly not a $Q$-group. On the other hand it is readily seen that groups satisfying the ascending chain condition for normal subgroups are $Q$-groups. Deeper is the fact that the group $G$ is a $Q$-group if it belongs to one of the following three classes of groups.

(a) Free groups on a finite number of generators. ${ }^{1}$

(b) Groups of finite dimensional matrices with coefficients from a field, which are generated by a finite number of elements. ${ }^{2}$

(c) Free products of a finite number of abelian groups each of which is generated by a finite number of elements. ${ }^{3}$

All these groups are generated by a finite number of elements. But we are able to show that this latter condition is neither necessary nor sufficient for a group to be a $Q$-group. The complexity of the situation is increased by the fact that neither subgroups nor quotient groups of $Q$-groups need be $Q$-groups. Thus it becomes desirable to obtain general criteria for a group to be a $Q$-group, and this is the main object of the present note.

The subgroup $S$ of the group $G$ shall be termed completely characteristic, if $S=S^{f}$ for every homomorphism $f$ of $G$ which satisfies $G=G^{f}$. Examples of completely characteristic subgroups are the commutator subgroup of $G$ and its generalizations. If $G$ happens to be a $Q$-group, then every characteristic subgroup of $G$ is completely characteristic.

Presented to the Society, April 29, 1944; received by the editors January 5, 1944.

${ }^{1}$ Levi [1]; Magnus [2]. The numbers in brackets refer to the bibliography at the end of the paper.

2 Malcew [1].

${ }^{3}$ Fouxe-Rabinowitch [1]. 
TheOREM 1. The group $G$ is a $Q$-group if there exists a well ordered ascending chain of completely characteristic subgroups $S_{v}$ with the following properties.

(i) $S_{0}=1 ; S_{k}=G$ for some $k$.

(ii) $S_{v+1} / S_{v}$ is a $Q$-group for every $v<k$.

(iii) If $v$ is a limit ordinal, then every element in $S_{v}$ is contained in some $S_{u}$ with $u<v$.

Proof. Suppose that $f$ is a homomorphism of $G$ satisfying $G=G^{f}$, and that $N$ is the kernel ${ }^{4}$ of this homomorphism $f$. We are going to prove by complete (transfinite) induction that the crosscut ${ }^{5}$ of $N$ and $S_{v}$ is 1 . Since this assertion is certainly true for $v=0$, we may assume its validity for every $u<v$.

Case 1. $v=u+1$ is not a limit ordinal.

Then we infer from our induction hypothesis that $N \cap S_{u}=1$. We deduce furthermore $S_{v}=S_{v}^{f}$ and $S_{u}=S_{u}^{f}$ from the fact that the subgroups $S$ are completely characteristic subgroups of $G$ and that $G=G^{f}$. Thus $f$ induces a homomorphism of $S_{v} / S_{u}$ such that $S_{v} / S_{u}=\left(S_{v} / S_{u}\right)^{\prime}$. But $S_{v} / S_{u}$ is by (ii) a $Q$-group and $f$ consequently induces an isomorphism in $S_{v} / S_{u}$. If $x$ is an element in the crosscut of $N$ and $S_{v}$, then $\left(S_{u} x\right)^{f}=S_{u}$. Hence $S_{u} x=S_{u}$ and $x$ is in $S_{u}$. But the crosscut of $S_{u}$ and $N$ is 1 , proving $x=1$. Thus $N \cap S_{v}=1$.

Case 2. $v$ is a limit ordinal.

If $x$ belongs to $S_{v} \cap N$, then $x$ belongs by (iii) to $S_{u} \cap N$ for $u<v$. Hence $x=1$ may be inferred from the induction hypothesis, showing again that $N \cap S_{v}=1$.

Thus it follows in particular that $N=N \cap G=N \cap S_{b}=1$, proving that $f$ is an isomorphism, as we desired to show.

The subgroup $S$ of the group $G$ has been termed strictly characteristic, ${ }^{6}$ if $S^{f} \leqq S$ for every homomorphism $f$ of $G$ satisfying $G^{f}=G$. The members $Z_{v}(G)$ of the ascending central chain of the group $G$ are strictly characteristic subgroups of $G$. To prove this it suffices to recall their inductive definition.

(i) $Z_{0}(G)=1$.

(ii) $Z_{v+1}(G) / Z_{v}(G)$ is the center ${ }^{7}$ of $G / Z_{v}(G)$.

(iii) If $v$ is a limit ordinal, then $Z_{v}(G)$ is the (set theoretical) join of the subgroups $Z_{u}(G)$ for $u<v$.

4 The kernel of the homomorphism $f$ consists of the elements in $G$ which are mapped upon 1 by $f$.

- The crosscut of the sets $A$ and $B$ will always be denoted by $A \cap B$.

- For a discussion of this and related concepts see Baer [2].

7 The center of the group $G$ consists of all the elements $z$ in $G$ which satisfy $z x=x z$ for every $x$ in $G$. 
Let us note that the group $G$ is termed nilpotent ${ }^{8}$ if there exists an ordinal $z$ such that $G=Z_{z}(G)$.

The members of the ascending central series, though strictly characteristic, need not be completely characteristic. This will be apparent from the following example, ${ }^{9}$ which shows at the same time the impossibility of substituting "strictly characteristic" for "completely characteristic" in Theorem 1.

Let $B$ be an abelian group of type $p^{\infty}$. Then $B$ contains one and only one subgroup $B_{i}$ which is a cyclic group of order $p^{i}$, and every element in $B$ is in at least one $B_{i}$. Denote by $G$ the group obtained by adjoining to $B$ an element $g$ subject to the relation

$$
g^{-1} x g=x^{1+p}
$$

for $x$ in $B$. It is readily seen that $B_{i}=Z_{i}(G)$ for finite $i$, that $B=Z_{\omega}(G)$ and that $G=Z_{\omega+1}(G)$. Thus $G$ is nilpotent and the quotient groups of successive terms in the ascending central series are cyclic groups (and therefore $Q$-groups). But $G$ is not a $Q$-group, since $G$ and $G / B_{1}$ are clearly isomorphic groups.

CoRollary. If $G$ is a nilpotent group and if every $Z_{v+1}(G) / Z_{v}(G)$ is $a$ a-group, then the following condition is necessary and sufficient for $G$ to be a Q-group.

Every $Z_{v}(G)$ is a completely characteristic subgroup of $G$.

The sufficiency of this condition is an immediate consequence of Theorem 1; its necessity may be derived from the fact that every characteristic subgroup of a $Q$-group is completely characteristic. The impossibility of omitting this condition from the statement of this corollary is a consequence of the considerations immediately preceding this corollary.

LEMMA. If $S$ is a strictly characteristic subgroup of the group $G$, and if $G / S$ is a $Q$-group, then $S$ is completely characteristic.

Proof. If $f$ is an endomorphism of $G$ such that $G=G^{f}$, then $S^{f} \leqq S$ and an endomorphism of $G / S$ upon itself is defined by mapping the coset $X$ in $G / S$ upon the coset $S X^{f}$ in $G / S$. Since $G / S$ is a $Q$-group, it follows that this endomorphism of $G / S$ is an automorphism. From this fact we conclude that the element $x$ in $G$ belongs to $S$ if, and only if, $x^{f}$ belongs to $S$. But to every element $y$ in $S$ there exists an element $z$ in $G$ such that $z^{f}=y$; and from the preceding remark we infer now that $z$ belongs to $S$ too, proving $S=S^{f}$, as we desired to show.

${ }^{8}$ See Baer [1] for a discussion of this concept of nilpotency.

- This is essentially a restatement of Baer [1, Example 2.5, p. 406]. 
The next criterion is an almost exact dual to Theorem 1 with the important difference that we need only consider strictly characteristic subgroups.

THEOREM 2. The group $G$ is a Q-group if there exists a well ordered descending chain of strictly characteristic subgroups $S_{v}$ with the following properties.

(i) $S_{0}=G ; S_{k}=1$ for some ordinal $k$.

(ii) $S_{v} / S_{v+1}$ is a $Q$-group for every $v<k$.

(iii) If $v$ is a limit ordinal, then $S_{v}$ is the crosscut of the subgroups $S_{u}$ for $u<v$.

Proof. If the homomorphism $f$ of the group $G$ satisfies $G=G^{f}$, then we are going to prove the following assertion by complete (transfinite) induction with respect to $v$.

(v) $S_{v}=S_{v}^{f}$ and $f$ induces an isomorphism of $G / S_{v}$.

Since this assertion $(u)$ certainly holds true for $u=0$, we may assume its validity for every $u<v$, in order to prove $(v)$. We distinguish two cases.

Case 1. $v=u+1$ is not a limit ordinal.

Then we infer from the induction hypothesis that $S_{u}=S_{u}^{f}$ and that $f$ induces an isomorphism in $G / S_{u}$. Since $S_{v}$ is strictly characteristic, we have $S_{v}^{f} \leqq S_{v}$. If $X$ is a coset in $S_{u} / S_{v}$, then mapping $X$ upon $S_{v} X^{f}$ effects a homomorphism of $S_{u} / S_{v}$ upon itself, that is, $S_{u} / S_{v}$ is the image of $S_{u} / S_{v}$. under this homomorphism. But $S_{u} / S_{v}$ is by (ii) a $Q$-group, and thus $f$ induces a proper automorphism of $S_{u} / S_{v}$. Suppose now that $X$ is a coset of $G / S_{v}$ which is mapped upon 1 by the homomorphism $f$ of $G / S_{v}$. Then $X^{f} \leqq S_{v}$ and therefore $S_{u} X^{f}=S_{u}$. But $f$ induces an isomorphism in $G / S_{u}$ so that $X \leqq S_{u}$ and $X$ is a coset of $S_{u} / S_{v}$. But then $X=1$, since $f$ induces an isomorphism in $S_{u} / S_{v}$, and we have shown that $f$ induces a proper automorphism of $G / S_{v}$. If $x$ is an element in $S_{v}$, then there exists an element $y$ in $G$ such that $x=y^{f}$. The coset $S_{v} y$ is mapped by $f$ into part of $S_{v}$. Since $f$ induces an isomorphism in $G / S_{v}$, as we have shown just now, it follows that $S_{v} y=S_{v}$, and this proves $S_{v}=S_{v}^{f}$, completing the proof of the case $(v)=(u+1)$.

Case 2. $v$ is a limit ordinal.

If $X$ is a coset of $G / S_{v}$ such that $X^{f} \leqq S_{v}$, then $X^{f} \leqq S_{u}$ for $u<v$. From the induction hypothesis we infer that $f$ induces an isomorphism in $G / S_{u}$ for $u<v$. Since $f$ maps the coset $S_{u} X$ of $G / S_{u}$ upon the identity of $G / S_{u}$, it follows that $X \leqq S_{u}$ for $u<v$, and we infer from (iii) that $X \leqq S_{v}$. Thus $f$ induces a proper automorphism of $G / S_{v}$; the fact that $S_{v}=S_{v}^{f}$ is verified as in Case 1 . 
Thus we have verified $(v)$ for every $v \leqq k$. From condition (i) and proposition $(k)$ we infer finally that $f$ is an automorphism of $G$, as we desired to show.

To enunciate two important consequences of this theorem we need the descending central series of $G$, whose members ${ }^{v} G$ are defined inductively as follows:

(i) ${ }^{\circ} G=G$.

(ii) ${ }^{v+1} G$ is generated by all the commutators $x^{-1} y^{-1} x y$ for $x$ in $G$ and $y$ in ${ }^{v} G$.

(iii) If $v$ is a limit ordinal, then ${ }^{v} G$ is the crosscut of all the ${ }^{u} G$ for $u<v$.

It is readily verified that ${ }^{v} G^{f} \leqq{ }^{v} G$ for every endomorphism ${ }^{10} f$ of $G$; that is, the subgroups ${ }^{v} G$ are fully invariant, ${ }^{11}$ and they are both completely and strictly characteristic subgroups of $G$. This descending central chain certainly meets the requirement (iii) of Theorem 2, and thus we obtain the following criterion.

Corollary 1. The group $G$ is a $Q$-group, if there exists an ordinal $k$ such that ${ }^{k} G=1$, and if the (abelian) groups ${ }^{v} G /{ }^{*+1} G$ are all $Q$-groups.

An abelian group is certainly a $Q$-group if it is generated by a finite number of elements. It is well known furthermore that ${ }^{i} G /{ }^{i+1} G$, for finite $i$, is generated by a finite number of elements if $G$ is generated by a finite number of elements. Thus the following fact is a special case of Corollary 1.

CoROLlaRY $2 .{ }^{12}$ The group $G$ is a $Q$-group if it is generated by a finite number of elements, and if ${ }^{\omega} G=1$.

If $F$ is a free group, then Magnus ${ }^{13}$ has shown that ${ }^{\omega} F=1$, and thus the last criteria are generalizations of the theorem ${ }^{14}$ that free groups on a finite number of generators are $Q$-groups.

In order to apply Theorem 2 to abelian groups we introduce some notations. If $A$ is an abelian group, then denote by $F_{p}(A)$ the set of all the elements in $A$ whose order is a power of the prime number $p$. Clearly $F_{p}(A)$ is a fully invariant subgroup of $A$. The product $F(A)$ of all the $F_{p}(A)$ is their direct product and consists of all the elements of finite order whereas $A / F(A)$ contains no elements of finite order except 1.

\footnotetext{
${ }^{10}$ Endomorphisms of the group $G$ are homomorphisms of $G$ into $G$.

${ }^{11}$ This concept is due to Levi [1]; for further discussion see Baer [2].

12 Fouxe-Rabinowitch [1].

13 Magnus [1].

${ }^{14}$ See footnote 1.
} 
Corollary 3. If $A / F(A)$ as well as every $F_{p}(A)$ is a Q-group, then the abelian group $A$ is a $Q$-group.

Proof. Let $S_{0}=A, S_{1}=F(A), S_{i}=\prod_{i \leqq j} F_{p_{j}}(A)$ where $p_{1}, p_{2}, \cdots$ is some enumeration of the primes. The subgroups $S_{i}$ are clearly strictly characteristic and their crosscut $S_{\omega}=1$. Since $S_{0} / S_{1}=A / F(A)$ and since $S_{i} / S_{i+1}$ is, for positive $i$, isomorphic to $F_{p_{i}}(A)$, the hypotheses of Theorem 2 are satisfied by this descending chain of subgroups, proving the validity of our present contention.

Let us note that $F_{p}(A)$ is a $Q$-group if it is finite, and that $F / F(A)$ is a $Q$-group if it is of finite rank, that is, if there exists an integer $k$ such that every subgroup of $F / F(A)$ which may be generated by a finite number of elements may also be generated by $k$ elements. Combining Corollaries 1 and 3 and the preceding remarks it is easy to construct any number of examples of $Q$-groups that cannot be generated by a finite number of elements.

Examples. 1. It is known that the free group on two generators contains a free group on an infinity of generators as a subgroup. ${ }^{15}$ The former group is a $Q$-group, the latter is not. This shows that not every subgroup of a $Q$-group need be a $Q$-group.

2. The additive group $R$ of the rational numbers cannot be generated by a finite number of elements. But $R$ is a $Q$-group. For if $R$ is considered modulo a subgroup different from 0 , then all the elements in the quotient group are of finite order. If $J$ is the subgroup of the integers in $R$, and if $E$ is the subgroup of the even integers in $R$, then $E \leqq J$ and $R / J$ and $R / E$ are isomorphic groups so that $R / E$ is not a $Q$-group, showing that quotient groups of $Q$-groups need not be Q-groups.

3. We are going to construct an example of a group $G$ with the following properties.

(a) $G$ is generated by two elements.

(b) $\left(G, G^{\prime \prime}\right)=1$.

(c) $G$ is not a $Q$-group.

Here we denote by $G^{\prime}$ the commutator subgroup of $G$, by $G^{\prime \prime}$ the commutator subgroup of $G^{\prime}$, and by $\left(G, G^{\prime \prime}\right)$ the subgroup generated by all the commutators $x^{-1} y^{-1} x y$ for $x$ in $G$ and $y$ in $G^{\prime \prime}$.

This example shows that Corollary 2 of Theorem 2 is in certain respects a "best" result.

To construct the group $G$ we proceed as follows. Let $F$ be a free group on two generators and denote by $u, v$ a free pair of generators of $F$. Then $F / F^{\prime}$ is the free abelian group of rank 2. The group $F^{\prime} / F^{\prime \prime}$

${ }^{16}$ For example, the commutator subgroup. 
may be shown to be a free abelian group, a basis of which is given by the elements

$$
(i, j)=u^{-i} v^{-j} u^{-1} v^{-1} u v v^{j} u^{i}
$$

for integral $i, j$. We put $N=\left(F, F^{\prime \prime}\right)$. Then $F^{\prime \prime} / N$ is part of the center of $F / N$; it is a free abelian group, generated by the elements (commutators)

$$
(i, j)\left(i^{\prime}, j^{\prime}\right)(i, j)^{-1}\left(i^{\prime}, j^{\prime}\right)^{-1} \text {. }
$$

Every subgroup of $F^{\prime \prime} / N$ is a normal subgroup of $F / N$, since $F^{\prime \prime} / N$ is part of the center of $F / N$. We denote by $U$ the subgroup of $F$ generated by $N$ and the elements $(0, j)\left(0, j^{\prime}\right)(0, j)^{-1}\left(0, j^{\prime}\right)^{-1}$ for all positive $j, j^{\prime}$, and we denote by $V$ the subgroup of $F$ generated by $N$ and all the elements $(0, j)\left(0, j^{\prime}\right)(0, j)^{-1}\left(0, j^{\prime}\right)^{-1}$ for $j, j^{\prime}$ not negative. It is clear that $U$ and $V$ are normal subgroups of $F$ and that

$$
N<V<U<F^{\prime \prime} \text {. }
$$

We are going to prove now that $G=F / V$ is the desired group. It is immediately clear that conditions (a) and (b) are satisfied by $G$. To prove (c) we construct an automorphism of the group $F$ which maps $V$ upon $U$. There exists one and only one automorphism $f$ of $F$ which maps $u$ upon $u v$ and $v$ upon $v$. We have

$$
F^{\prime f}=F^{\prime}, \quad F^{\prime \prime}=F^{\prime \prime}, \quad N^{f}=N,
$$

since these subgroups are characteristic subgroups of $F$. Furthermore we have

$$
(0,0)^{f}=\left(u^{-1} v^{-1} u v\right)^{f}=(u v)^{-1} v^{-1}(u v) v=v^{-1}(0,0) v=(0,1),
$$

and consequently

$$
(0, j)^{f}=(0, j+1)
$$

for integral $j$. From this last equation one derives the equality $V^{f}=U$, completing the proof.

From the preceding arguments one deduces readily the noteworthy fact that the ascending chain condition is not satisfied by the normal subgroups of a nonabelian free group.

Dualizing the concept of a $Q$-group we define as an $S$-group a group $G$ meeting the following requirement.

(S) If the subgroup $S$ of $G$ is isomorphic to $G$, then $S=G$.

It is readily seen that every group satisfying the descending chain condition for subgroups is an $S$-group. An example of an $S$-group not satisfying this chain condition is the additive group of rational numbers. 
To enunciate conveniently a criterion for $S$-groups we need the following concept. The subgroup $C$ of the group $G$ is said to be an $S$-characteristic subgroup of $G$ if $C^{f} \leqq C$ for every isomorphism $f$ of $G$ into itself. Clearly every fully invariant subgroup is $S$-characteristic and every $S$-characteristic subgroup is characteristic. If $G$ happens to be an $S$-group, then conversely every characteristic subgroup of $G$ is $S$-characteristic.

THEOREM 3. The group $G$ is an $S$-group if there exists a well ordered ascending chain of S-characteristic subgroups $N(v)$ of $G$ with the following properties.

(i) $N(0)=1$ and $N(t)=G$ for some ordinal $t$.

(ii) $N(v+1) / N(v)$ is an $S$-group.

(iii) If $v$ is a limit ordinal, then every element in $N(v)$ is contained in some $N(u)$ for $u<v$.

Proof. Suppose that $f$ is an isomorphism of $G$ upon some subgroup $S$ of $G$. We are going to prove by complete (transfinite) induction that $N(v)^{f}=N(v)$ for every $v$. This assertion is certainly true for $v=0$; and thus we may assume its validity for every $u<v$. If $v$ happens to be a limit ordinal, then our contention is directly derived from (iii) and the induction hypothesis. If, however, $v=u+1$ is not a limit ordinal, then we deduce from $N(u)^{f}=N(u)$ and $N(v)^{f} \leqq N(v)$ that $f$ induces an isomorphism of the group $N(v) / N(u)$ upon a subgroup of $N(v) / N(u)$. But it follows from (ii) that $N(v) / N(u)$ is an $S$-group. Hence $(N(v) / N(u))^{f}=N(v) / N(u)$ or $N(v)=N(v)^{f} N(u)=N(v)^{f} N(u)^{f}$ $=N(v)^{f}$, as we desired to show. Now $G=N(t)=N(t)^{f}=G^{f}$ is a consequence of (i).

We are interested in the relations between $Q$-groups and $S$-groups. There are groups that are both, like the finite groups or the additive group of rational numbers. Any direct (or free) product of an infinity of isomorphic groups $(\neq 1)$ is neither. The infinite cyclic groups are $Q$-groups, but not $S$-groups, and the abelian groups of type $p^{\infty}$ are $S$-groups, but not $Q$-groups. Thus the following theorem seems to be of some interest.

THEOREM 4. If $N$ is a fully invariant subgroup of the free group $F$, and if $G=F / N$ is an $S$-group, then $G$ is a $Q$-group.

Proof. If $L$ is a normal subgroup of $G$ such that $G$ and $G / L$ are isomorphic groups, then denote by $M$ the uniquely determined normal subgroup of $F$ such that $N \leqq M$ and $M / N=L$. There exists an isomorphism $g$ of $G$ upon $G / L$, and hence there exists an endomorphism $f$ of $F$ satisfying $X^{g}=M X^{f}$ for every coset $X$ of $F / N=G$. (To 
prove the existence of $f$, consider a free set of generators of $F$ and define $f$ on this free set of generators in accordance with our requirement. Then $f$ can easily be seen to be a desired endomorphism.) Then $F=F^{f} M$ and $F^{f} \cap M=N^{f} \leqq N$, since $N$ is a fully invariant subgroup of $F$. Since $M$ and $N$ are normal subgroups of $F$, we deduce from Dedekind's law that

$$
F^{f} N \cap M=\left(F^{f} \cap M\right) N=N^{f} N=N,
$$

and from $N \leqq M$ we infer $\left(F^{f} N\right) M=F^{\prime} M=F$. The subgroup $\left(F^{f} N\right) / N$ $=V$ of $G=F / N$ has therefore the following properties:

$$
V \cap L=1 \text { and } V L=G .
$$

Thus every coset of $G / L$ contains one and only one element of the subgroup $V$ of $G$, proving that $V$ and $G / L$ are isomorphic. But $G / L$ and $G$ are isomorphic and $G$ is an $S$-group, proving that $V=G$. This implies finally $L=1$, showing that $G$ is a $Q$-group.

It is apparent from the proof of this theorem that it remains valid if we substitute for the hypothesis that $F$ be a free group the following assumption:

If $M$ and $N$ are normal subgroups of $F$, if $N \leqq M$, and if $g$ is an isomorphism of $F / N$ upon $F / M$, then there exists an endomorphism $f$ of $F$, satisfying

$$
X^{g}=M X^{f}
$$

for every coset $X$ of $F / N$.

It has been shown that the properties (1) to (3) may be deduced (a) from the existence of an ascending chain of completely characteristic subgroups, meeting certain requirements (Theorem 1), and (b) from the existence of a descending chain of strictly characteristic subgroups, satisfying certain conditions (Theorem 2). But these properties (a) and (b) are not independent. For the following correspondence between subgroups maps every ascending chain of completely characteristic subgroups upon a descending chain of strictly characteristic subgroups. If $S$ is a subgroup of the group $G$, then let $S^{*}$ be the centralizer of $S$ in $G$, which consists exactly of those elements in $G$ commuting with all the elements in $S$. To substantiate our contention we enumerate a number of properties of this operation.

(1) If $S \leqq T$, then $T^{*} \leqq S^{*}$.

(2) If $S$ is the (set theoretical) join of the subgroups $S_{v}$, then $S^{*}$ is the crosscut of the subgroups $S_{v}^{*}$.

These two properties are immediately obvious. It should be noted, however, that neither the dual nor the converse of (2) is true. 
(3) If $S$ is a completely characteristic subgroup of $G$, then $S^{*}$ is a strictly characteristic subgroup of $G$.

Proof. If $G=G^{f}$ is satisfied by the endomorphism $f$ of $G$, if $s$ is an element in $S$, and if $t$ is an element in $S^{*}$, then there exists an element $s^{\prime}$ in $S$ such that $s=s^{\prime f}$; and we find:

$$
s t^{f}=s^{\prime f} t^{f}=\left(s^{\prime} t\right)^{f}=\left(t s^{\prime}\right)^{f}=t^{\prime} s^{\prime f}=t^{\prime} s,
$$

proving that $t^{f}$ belongs to $S^{*}$ too, or $S^{* f} \leqq S^{*}$.

REMARK. The impossibility of proving that $S^{*}$ is completely characteristic, if $S$ is completely characteristic, may be shown by the following example: If $S=G$, then $S$ is certainly completely characteristic. But $S^{*}$ is the center of $G$; and we have pointed out before that the center is not always completely characteristic.

Note that in the proof of (3) we actually verified the following fact. If $S$ is a subgroup of $G$, if $f$ is an endomorphism of $G$, and if $S \leqq S^{f}$, then $S^{* f} \leqq S^{*}$. This implies in particular:

(4) $S^{*}$ is a normal subgroup of $G$, if $S$ is a normal subgroup of $G$.

To enunciate the next statement it will be convenient to introduce some notations. If $X$ and $Y$ are subsets of $G$, then we denote by $(X, Y)$ the subgroup generated by all the commutators $x^{-1} y^{-1} x y$ for $x$ in $X$ and $y$ in $Y$. For these subgroups $\mathrm{P}$. Hall proved the following important inequality:

$(\mathrm{H})^{16}$ If $A, B, C$ are normal subgroups of $G$, then

$$
(A,(B, C)) \leqq(B,(C, A))(C,(A, B)) .
$$

Now we are able to prove

(5) If $(G, T) \leqq S$ is satisfied by the subgroups $S$ and $T$ of $G$, then $\left(S^{*}, S^{*}\right) \leqq T^{*}$.

Proof. We deduce from $(\mathrm{H})$ that

$\left(T,\left(S^{*}, S^{*}\right)\right) \leqq\left(S^{*},\left(S^{*}, T\right)\right) \leqq\left(S^{*},(G, T)\right) \leqq\left(S^{*}, S\right)=1$, proving $(5)$.

We define the derived series $G^{(v)}$ of the group $G$ inductively as follows.

(i) $G^{(0)}=G$.

(ii) $G^{(v+1)}$ is the commutator subgroup of $G^{(v)}$.

(iii) If $v$ is a limit ordinal, then $G^{(v)}$ is the crosscut of the subgroups $G^{(u)}$ for $u<v$.

If in particular there exists an ordinal $s$ such that $G=G^{(s)}$, then $G$ may be termed soluble. ${ }^{17}$

\footnotetext{
${ }^{16}$ Hall [1].

${ }^{17}$ A different concept of solubility which is narrower than the present one has been discussed in Baer [1].
} 


\section{Theorem 5. Nilpotent groups are soluble.}

Proof. Put $C_{v}=Z_{v}(G) *$ for every $v$. Then we have $C_{0}=C_{1}=G$, since $Z_{1}(G)$ is the center of $G$. From $\left(G, Z_{v+1}(G)\right) \leqq Z_{v}(G)$ and property (5) we deduce that

$$
\left(C_{v}, C_{v}\right) \leqq C_{v+1}
$$

If $v$ is a limit ordinal, then $Z_{v}(G)$ is the join of the $Z_{u}(G)$ for $u<v$, and we infer from (2) that $C_{v}$ is the crosscut of the subgroups $C_{u}$ for $u<v$. Hence it follows by complete (transfinite) induction that

$$
G^{(v)} \leqq C_{v} \text { for every } v \text {. }
$$

Since $G$ is nilpotent, there exists an ordinal $k$ such that $G=Z_{k}(G)$. Clearly $C_{k}$ is the center of $G$. But $G^{(k)}$ is part of $C_{k}$ and $C_{k}$ is abelian. Hence $G^{(k+1)}=1$, proving the solubility of $G$.

Appended to Theorem 1 is a discussion of an example of a nilpotent group $G$ which can be shown to have the property ${ }^{1} G={ }^{2} G \neq 1$. Thus nilpotency does not imply that the descending central series ends with 1.

On the other hand it may be worth noting that the nonabelian free groups $F$ satisfy ${ }^{\omega} F=1$, though their center is equal to 1 , showing the impossibility of deducing nilpotency from the fact that the descending central chain ends with 1.

We note finally that the situation is altogether different if one is mainly interested in finite chains. For the following theorem is well known: ${ }^{18} G=Z_{n}(G)$ for finite $n$ if, and only if, $1={ }^{m} G$ for finite $m$.

R. BAER

\section{BIBLIOGRAPHY}

1. Nilpotent groups and their generalizations, Trans. Amer. Math. Soc. vol. 47 (1940) pp. 393-434.

2. The higher commutator subgroups of a group, Bull. Amer. Math. Soc. vol. 50 (1944) pp. 143-160.

D. I. FouXE-RABINOWITCH

1. On the determinators of an operator of the free group, Rec. Math. (Mat. Sbornik) N.S. vol. 7 (1940) pp. 197-206.

P. HALL

1. A contribution to the theory of groups of prime-power order, Proc. London Math. F. LEVI Soc. vol. 36 (1932) pp. 29-95.

1. Über die Untergruppen der freien Gruppen, Math. Zeit. vol. 37 (1933) pp. 90-97. W. MAGNUS

1. Beziehungen zwischen Gruppen und Idealen in einem speziellen Ring, Math. Ann. vol. 111 (1935) pp. 259-280.

18 Hall [1] or Baer [1, footnote on p. 406]. 
2. Über freie Faktorgruppen und freie Untergruppen gegebener Gruppen, Monatshefte für Mathematik und Physik vol. 47 (1938) pp. 307-313.

A. Malcew

1. On isomorphic matrix representations of infinite groups, Rec. Math. (Mat. Sbornik) vol. 8 (1940) pp. 421-422 (405-421).

UNIVERSITY OF ILLINOIS

\section{A SUBSTITUTE FOR THE AXIOM OF CHOICE}

\section{A. D. WALlace}

The following result appeared in the 1914 edition of Hausdorff's Mengenlehre, p. 140:

(A) Any partially ordered system contains a maximal simply ordered subsystem.

This theorem is well known to be equivalent to the axiom of choice (though there does not seem to be a proof of this fact in the literature) and it has been suggested as an alternative for this axiom. The purpose of this note (which is purely methodological) is to propose a simpler but equivalent formulation of (A) as a substitute for the Zermelo axiom. The simplicity lies in the fact that we make no assumptions concerning the relation $R$ which replaces partial order.

Let $Q$ be a set and $R$ an arbitrary binary relation on $Q$. A subset of $Q$ will be termed $R$-simple if for any pair of its elements, $a$ and $b$, we have either $a R b$ or $b R a$. The version of (A) we propose is:

(B) Any R-simple subset of $Q$ is contained in a maximal $R$-simple subset of $Q$.

It is clear that (B) implies (A). Conversely, let $Q_{0}$ be an $R$-simple subset of $Q$. Let $P$ be the partially ordered (by inclusion) system composed of all $R$-simple subsets of $Q$ which contain $Q_{0}$. Then by (A) there is a maximal simply ordered subsystem $P_{0}$ of $P$. The union of all the sets in $P_{0}$ is the desired maximal $R$-simple subset.

The University of Pennsylvania

Received by the editors December 12, 1943. 